Document downloaded from:

http://hdl.handle.net/10251/43472

This paper must be cited as:

Estrada, H.; Gómez Lozano, V.; Uris Martínez, A.; Candelas Valiente, P.; Belmar Ibáñez, F.; Meseguer Rico, FJ. (2011). Sound transmission through plates perforated with two periodic subwavelength hole arrays. Journal of Physics: Condensed Matter. 23(135401). doi:10.1088/0953-8984/23/13/135401.

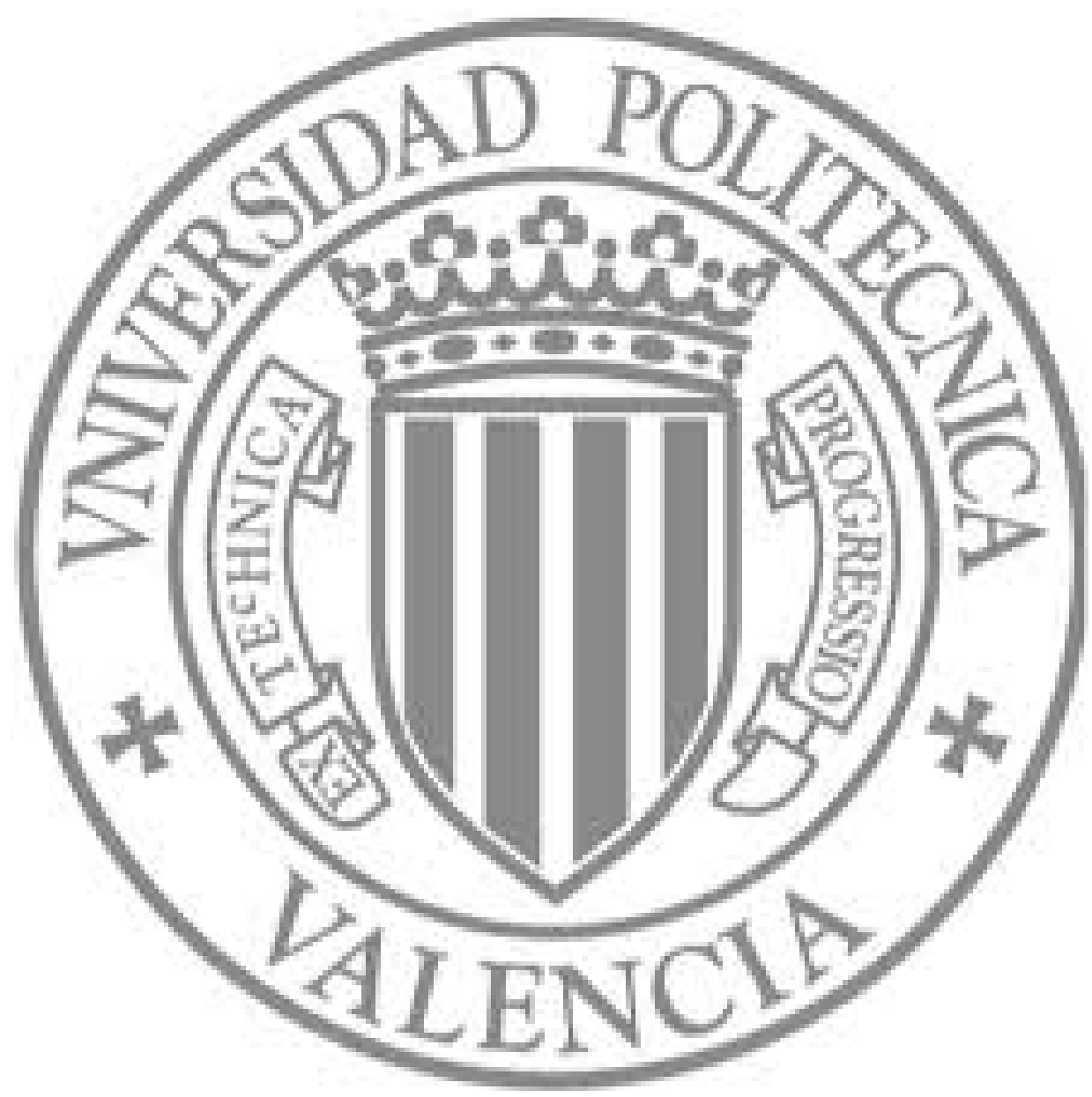

The final publication is available at

http://dx.doi.org/10.1088/0953-8984/23/13/135401

Copyright Institute of Physics Publishing (IOP) 


\title{
SOUND TRANMISSION THROUGH PLATES PERFORATED WITH TWO PERIODIC SUBWAVELENGTH HOLE ARRAYS
}

Héctor Estrada, Vicente Gómez-Lozano, Antonio Uris ${ }^{1}$, Pilar Candelas, Francisco Belmar, Francisco Meseguer

Centro de Tecnologías Físicas, Unidad Asociada ICMM-CSIC/UPV, Universidad Politécnica de Valencia, Av. Naranjos s/n. 46022 Valencia, Spain Phone: 34.963877528; Fax: 34.963879525; E-mail: auris@fis.upv.es

${ }^{1}$ Corresponding author.

\begin{abstract}
Theoretical results of sound transmission through plates perforated with compound periodic hole arrays are presented. Calculations are obtained by means of a model under the rigid-solid assumption, which is thoroughly derived. A complex interplay between resonant transmission peaks, resonance interference, and Wood anomaly minima is observed. At high frequencies the resonant peaks overcome the Wood anomaly dips, leading to a different behaviour when compared with simple hole arrays.
\end{abstract}

Keywords: Subwavelength, transmission band, hole array, periodicity.

\section{INTRODUCTION}

In the last years, extraordinary optical transmission through metallic films perforated with subwavelength apertures [1] has received great attention, not only for 
the rich physics behind the phenomenon [2-4], but also because of its potential application in optics [5-8]. Several phenomena are proposed to be involved into the extraordinary optical transmission, such as surface plasmon resonances [9, 10], waveguide resonance [2], cavity resonances [4] and dynamical diffraction [3]. Recently, the ideas initially developed for electromagnetic waves have been extended to the field of acoustics, although some differences make the acoustical case unique, i.e. acoustic waves can be transmitted through a subwavelength aperture [11] and sound can penetrate into the solid [19]. Acoustic transmission through subwavelength apertures has been investigated both theoretically and experimentally. Complete transmission through two dimensional hole arrays was predicted by Zhou and Kriegsman [12]. Experimental verification of complete transmission for slit and hole arrays was reported by $\mathrm{Lu}$ et al. [13] and Hou et al. [14], respectively. It was confirmed that the main contribution to the full transmission peaks were Fabry-Perot resonances in the holes. Theoretical results for one and two dimensional arrays of square holes were reported by Christensen et al. [15]. Estrada et al. [16] reported that periodically perforated aluminium plates immersed in water can achieve lower ultrasonic transmission values than homogeneous plates. The influence of the filling fraction of holes and the lattice geometry in the transmission properties has also been studied $[17,18]$. It has been also demonstrated [19] that the complex interplay between Fabry-Perot resonances inside the holes, lattice resonances in periodic arrays, and elastic Lamb modes governs the transmission properties of perforated plates. Liu and Jin [20] have predicted the existence of narrow dips within full transmission peaks due to phase resonances when the hole array unit cell is asymmetric.

The aim of this paper is to analyse theoretically the sound transmission through plates perforated with compound hole arrays resulting from two periodic arrays with a rigid 
solid model approximation. First, the model used to calculate sound transmission is presented and followed by a second part dedicated to analyse the numerical results.

\section{THEORY}

Figure 1 shows schematically a plate of thickness $h$ drilled with $P$ cylindrical holes of radius $r_{0}^{i}$ in positions determined by their centres $\vec{r}_{i}$.

Supposing an incident plane wave $\phi_{0}(\vec{r})=e^{i\left(\vec{k}_{0} \cdot \vec{r}-\omega t\right)}$, the reflected and the transmitted sound pressure fields are plane-wave expanded. For simplicity, time harmonic excitation is assumed, thus the time component $e^{-i \omega t}$ can be omitted. Under the rigid-solid assumption, there is no field inside the solid. The pressure field in the three regions can be written as follows [21]:

$$
\begin{aligned}
& \phi_{I}(\vec{r})=\phi_{0}(\vec{r})+\phi_{R}(\vec{r})=e^{i\left(\vec{Q}_{0} \cdot \vec{r}-q_{0} z\right)}+\iint \beta^{+}(\vec{Q}) e^{i(\vec{Q} \cdot \vec{r}+q z)} d^{2} \vec{Q}, \\
& \phi_{I I}(\vec{r})=\left\{\begin{array}{ll}
\sum_{i=1}^{P} \sum_{m=0}^{\infty} \sum_{n=1}^{\infty} J_{m}\left(Q_{m n}^{i}\left|\left(\vec{r}-\vec{r}_{i}\right)_{\|}\right|\right) e^{i m \varphi_{i}} \Psi_{m n}^{i}(z) \text { if }\left|\vec{r}-\vec{r}_{i}\right| \leq r_{0}^{i} \\
0 \quad \text { otherwise }
\end{array},\right. \\
& \phi_{I I I}(\vec{r})=\iint \beta^{-}(\vec{Q}) e^{i\left(\vec{Q} \cdot \vec{r}_{i}-q(z+h)\right)} d^{2} \vec{Q},
\end{aligned}
$$

where $\vec{k}_{0}=\left(\vec{Q}_{0}, q_{0}\right), k_{0}=\omega / c, q=\sqrt{k_{0}^{2}-Q^{2}}, q_{m n}^{i}=\sqrt{k_{0}^{2}-Q_{m n}^{i}}, J_{m}(\ldots)$ is the Bessel function of the first kind and order $\mathrm{m}$, and $\Psi_{m n}^{i}(z)$ is defined as $\Psi_{m n}^{i}(z)=\alpha_{m n}^{i+} e^{i q_{m n}^{i} z}+\alpha_{m n}^{i-} e^{-i q_{m n}^{i} z}$

Under the rigid-solid assumption, zero normal velocity at the hole walls is assumed and the polar eigenfunctions inside the hole must satisfy $J_{m}^{\prime}\left(Q_{m n}^{i} r_{0}^{i}\right)=0$. 
Repeating the $\mathrm{P}$ holes periodically throughout the whole plate, they can be considered as a lattice basis with a unit-cell area $\mathrm{S}$ and defined by the vectors $\left(\vec{a}_{1}, \vec{a}_{2}\right)$. In this way, the coefficients $\beta^{ \pm}(\vec{Q})$ can be expanded as Fourier series, giving discrete expressions [22] for equations (1) and (3)

$$
\begin{gathered}
\phi_{I}(\vec{r})=2 e^{i\left(\vec{Q}_{0} \cdot \vec{r}_{i}\right)} \cdot \cos \left(q_{0} \cdot z\right)+\sum_{\vec{G}} \beta_{\vec{G}}^{+} e^{i\left(\vec{Q}_{\vec{G}} \cdot \vec{r}_{\|}+q_{\vec{G}} z\right)}, \\
\phi_{I I I}(\vec{r})=\sum_{\vec{G}} \beta_{\vec{G}}^{-} e^{i\left(\vec{Q}_{\vec{G}} \cdot \vec{r}_{\|}-q_{\vec{G}}(z+h)\right)}
\end{gathered}
$$

where $\vec{Q}_{\vec{G}}=\vec{Q}_{0}+\vec{G}, \vec{G}$ is the reciprocal lattice vector of $\left(\vec{a}_{1}, \vec{a}_{2}\right)$, and $q_{\vec{G}}=\sqrt{k_{0}^{2}-Q_{\vec{G}}{ }^{2}}$.

The objective is to determine the coefficients $\beta_{\vec{G}}^{+}, \beta_{\vec{G}}^{-}, \alpha_{m n}^{i+}$ and $\alpha_{m n}^{i-}$ by imposing continuity conditions of pressure and normal velocity at the plate interfaces.

The continuity of the normal velocity at $z=0$ and $z=-h$ yields

$$
\begin{gathered}
\beta_{\vec{G}}^{+}=\frac{1}{S} \sum_{i=1}^{P} \sum_{m=0}^{\infty} \sum_{n=1}^{\infty} \frac{q_{m n}^{i}}{q_{\vec{G}}} I_{m n}^{i}\left(\vec{Q}_{\vec{G}}\right) \Psi_{m n}^{\prime i}(0), \\
\beta_{\vec{G}}^{-}=-\frac{1}{S} \sum_{i=1}^{P} \sum_{m=0}^{\infty} \sum_{n=1}^{\infty} \frac{q_{m n}^{i}}{q_{\vec{G}}} I_{m n}^{i}\left(\vec{Q}_{\vec{G}}\right) \Psi_{m n}^{\prime i}(-h),
\end{gathered}
$$

where

$$
\begin{aligned}
& \Psi_{m n}^{\prime i}(z)=\alpha_{m n}^{i+} e^{i q_{m n} z}-\alpha_{m n}^{i-} e^{-i q_{m n} z}=\frac{i}{q_{m n}^{i}} \frac{\partial \Psi_{m n}^{i}}{\partial z}, \\
& I_{m n}^{i}\left(\vec{Q}_{\vec{G}}\right)=\iint_{S_{i}} J_{m}\left(Q_{m n}^{i} \mid\left(\vec{r}-\vec{r}_{i}\right)_{\|}\right) e^{i m \varphi_{i}} e^{-i \vec{Q}_{\vec{G}} \cdot \vec{r}} d S=e^{-i \vec{Q}_{\vec{G}} \cdot \vec{r}_{i}} \int_{0}^{2 \pi} \int_{0}^{r_{0}^{i}} J_{m}\left(Q_{m n}^{i} r\right) e^{i m \varphi} e^{-i \vec{Q}_{\vec{G}} \cdot \vec{r}} r d r d \varphi,
\end{aligned}
$$

which integrates as

$$
I_{m n}^{i}\left(\vec{Q}_{\vec{G}}\right)=2 \pi i^{m} e^{-i \vec{Q}_{\vec{G}} \cdot \vec{r}_{i}} e^{i m \varphi_{G}}\left\{\begin{array}{l}
\frac{\left|\vec{Q}_{\vec{G}}\right| r_{0}^{i}}{\left|\vec{Q}_{\vec{G}}\right|^{2}-\left(Q_{m n}^{i}\right)^{2}} J_{m}\left(Q_{m n}^{i} r_{0}^{i}\right) J_{m}^{\prime}\left(\left|\vec{Q}_{\vec{G}}\right| r_{0}^{i}\right) \quad \text { if } \quad Q_{m n}^{i} \neq\left|\vec{Q}_{\vec{G}}\right|, \\
\frac{\left(r_{0}^{i}\right)^{2}}{2} J_{m}^{2}\left(Q_{m n}^{i} r_{0}^{i}\right)\left(1-\frac{m^{2}}{\left(Q_{m n}^{i} r_{0}^{i}\right)^{2}}\right) \quad \text { if } \quad Q_{m n}^{i}=\left|\vec{Q}_{\vec{G}}\right|,
\end{array}\right.
$$


where

$$
\varphi_{G}=\arcsin \left(Q_{\vec{G} y} / Q_{\vec{G} x}\right) .
$$

On the other hand, imposing pressure continuity at $z=0$, multiplying by $J_{m^{\prime}}\left(Q_{m^{\prime} n^{\prime}}^{j}\left|\left(\vec{r}-\vec{r}_{j}\right)_{\|}\right|\right) e^{-i m \varphi_{j}}$ integrating at the unit cell, considering the orthogonality of the exponential and the Bessel functions [23], and using equation (6) yields

$\overline{2 I_{m^{\prime} n^{\prime}}^{j}\left(\vec{Q}_{0}\right)}=-\frac{1}{S} \sum_{i=1}^{P} \sum_{m=0}^{\infty} \sum_{n=1}^{\infty} q_{m n}^{i} \Psi_{m n}^{\prime i}(0) M_{m m^{\prime} n n^{\prime}}^{i j}\left(\vec{Q}_{\vec{G}}\right)+\sum_{i=1}^{P} \sum_{m=0}^{\infty} \sum_{n=1}^{\infty} 2 \pi \delta_{i j} \delta_{m m^{\prime}} \delta_{n n^{\prime}} P_{m n}^{i} \Psi_{m n}^{i}(0)$,

where $2 \overline{I_{m^{\prime} n^{\prime}}^{j}\left(\vec{Q}_{0}\right)}$ is the complex conjugate of $2 I_{m^{\prime} n^{\prime}}^{j}\left(\vec{Q}_{0}\right)$,

$M_{m m^{\prime} n n^{\prime}}^{i j}\left(\vec{Q}_{\vec{G}}\right)=\sum_{\vec{G}} \frac{I_{m n}^{i}\left(\vec{Q}_{\vec{G}}\right) \overline{I_{m^{\prime} n^{\prime}}^{j}\left(\vec{Q}_{\vec{G}}\right)}}{q_{\vec{G}}}$,

and

$P_{m n}^{i}=\int_{0}^{r_{0}^{i}} J_{m}^{2}\left(Q_{m n}^{i}\left|\left(\vec{r}-\vec{r}_{i}\right)_{\|}\right|\right) r d r$

being this last integral analytically solvable [23]. Proceeding in the same way for pressure continuity at $z=-h$, but using equation (7) yields

$$
0=\frac{1}{S} \sum_{i=1}^{P} \sum_{m=0}^{\infty} \sum_{n=1}^{\infty} q_{m n}^{i} \Psi_{m n}^{\prime i}(-h) M_{m m^{\prime} n n^{\prime}}^{i j}\left(\vec{Q}_{\vec{G}}\right)+\sum_{i=1}^{P} \sum_{m=0}^{\infty} \sum_{n=1}^{\infty} 2 \pi \delta_{i j} \delta_{m n^{\prime}} \delta_{n n^{\prime}}{ }_{m n}^{i} \Psi_{m n}^{i}(-h)
$$

Truncating the series in $m=M$ and $n=N$ and naming

$$
\begin{aligned}
& A_{m m^{\prime} n n^{\prime}}^{i j}=2 \pi \delta_{i j} \delta_{m m^{\prime}} \delta_{n n^{\prime}} P_{m n}^{i}-\frac{1}{S} q_{m n}^{i} M_{m m^{\prime} n n^{\prime}}^{i j}, \\
& B_{m m^{\prime} n n^{\prime}}^{i j}=2 \pi \delta_{i j} \delta_{m m^{\prime}} \delta_{n n^{\prime}} P_{m n}^{i}+\frac{1}{S} q_{m n}^{i} M_{m m^{\prime} n n^{\prime}}^{i j}, \\
& C_{m m^{\prime} n n^{\prime}}^{i j}=B_{m m^{\prime} n n^{\prime}}^{i j} \cdot e^{-i q_{m n}^{i} h}, \\
& D_{m m^{\prime} n n^{\prime}}^{i j}=A_{m m^{\prime} n n^{\prime}}^{i j} \cdot e^{i q_{m n}^{i} h},
\end{aligned}
$$

the equations (8) and (9) become in a system of $2 \times M \times N \times P$ linear equations in the variables $\alpha_{m n}^{i+}$ and $\alpha_{m n}^{i-}$ 


$$
\begin{aligned}
& \sum_{i=1}^{P} \sum_{m=0}^{M-1} \sum_{n=1}^{N}\left(A_{m m^{\prime} n n^{\prime}}^{i j} \cdot \alpha_{m n}^{i+}+B_{m m^{\prime} n n^{\prime}}^{i j} \cdot \alpha_{m n}^{i-}\right)=2 \overline{I_{m^{\prime} n^{\prime}}^{j}\left(\vec{Q}_{0}\right)} \\
& \sum_{i=1}^{P} \sum_{m=0}^{M-1} \sum_{n=1}^{N}\left(C_{m m^{\prime} n n^{\prime}}^{i j} \cdot \alpha_{m n}^{i+}+D_{m m^{\prime} n n^{\prime}}^{i j} \cdot \alpha_{m n}^{i-}\right)=0 .
\end{aligned}
$$

Once $\alpha_{m n}^{ \pm}$are obtained, $\beta_{\vec{G}}^{ \pm}$can be calculated directly from equations (6) and (7).

The sound power radiated by an infinite plate is given by the expression [24]

$\Pi(\omega)=\frac{\rho c k_{0}}{8 \pi^{2}} \operatorname{Re}\left\{\iint_{-\infty}^{\infty} \frac{|V(\vec{Q})|^{2}}{\sqrt{k_{0}^{2}-Q^{2}}} d^{2} Q\right\}$,

where $V(\vec{Q})$ is the 2-dimensional Fourier spatial transform of the normal velocity $\frac{i}{\omega \rho} \frac{\partial \phi}{\partial z}$ on each side of the plate, then, the sound power transmission coefficient is calculated as $\tau=\frac{\Pi_{T}(\omega)}{\Pi_{0}(\omega, \theta, \varphi)}=\sum_{\vec{G}} \operatorname{Re}\left\{\frac{q_{\vec{G}}}{q_{0}}\right\}\left|\beta_{\vec{G}}^{-}\right|^{2}$.

\section{NUMERICAL RESULTS AND DISCUSSION}

The numerical calculations are made considering square and a rectangular compound hole arrays as described in Fig. 2. The compound hole arrangement consists in a primary square array which is decorated with a larger secondary array that can be square (Fig. 2(a)) or rectangular (Fig. 2(b)). The symmetry of the resulting structure is given by the symmetry of the secondary array. We can explore the transmission properties of compound arrays by defining three different samples, namely sample A having $d_{1}=2 \mathrm{~mm}, d_{2}=3 \mathrm{~mm}, a=8 \mathrm{~mm}$, and $\mathrm{h}=3 \mathrm{~mm}$; sample $B$ having $\mathrm{d}_{1}=2 \mathrm{~mm}, \mathrm{~d}_{2}=4$ $\mathrm{mm}, \mathrm{a}=10 \mathrm{~mm}$, and $\mathrm{h}=5 \mathrm{~mm}$; and sample $C$ with $\mathrm{d}_{1}=2 \mathrm{~mm}, \mathrm{~d}_{2}=3 \mathrm{~mm}, \mathrm{a}_{1}=12 \mathrm{~mm}, \mathrm{a}_{2}=8$ $\mathrm{mm}$, and $\mathrm{h}=3 \mathrm{~mm}$. Calculating transmitted sound power coefficient, $\tau$, as a function of 
wavelength, $\lambda$, in the fluid at normal incidence we obtain Fig. 3 . The contribution of the primary (black) and the secondary (gray) is calculated separately for samples A (Fig. 3(a)) and B (Fig. 3(c)). Although Fabry-Perot hole resonances, which are mainly governed by the plate thickness, have a major effect in the appearance of full transmission peaks, the hole size and the period can shift considerably the peak position [17]. The transmission of the compound array is depicted in Figs. 3(b) and 3(d) for samples A and B respectively. At a first sight could seem that the compound array transmission curve is simply the addition of the primary and secondary array curves. In fact, the three transmission peaks provided by the simple arrays occur almost at the same wavelengths than for the compound arrays. However, as pointed out in [20], destructive interference occurs between two transmission peaks leading to zero transmission. One remarkable feature of compound arrays is the existence of full transmission peaks for wavelengths smaller than the unit cell period. This property has not been observed for simple hole arrays so far. In addition, the first order full transmission peak of the primary array (Fig. 3(b)) prevails over the Wood anomaly [25] minima of the secondary (compound) array at $\lambda=8 \mathrm{~mm}$.

In contrast to the simplicity found in [20] when studying the angular dependency of $\tau$, a complex interplay between Wood anomaly minima, resonant interference minima and resonant full transmission is observed in the transmission dispersion plots from Fig. 4. Again samples A (Fig. 4(a) and (b)) and B (Fig. 4(c) and (d)) are studied. Plots at the right are the same than those at the left with the inclusion of Wood anomaly curves of the primary (white) and secondary array (black) to distinguish them from the resonant interference minima. The prevalence of the transmission peaks over the Wood anomaly minima is evident also when the incidence angle is varied. For both samples, the transmission maxima originated by the secondary array (first order Fabry-Perot) are 
flanked by the Wood minima whereas the first order transmission peak provided by the primary array is nearly flat. This can explain the angle dependence of the resonance interference which is different from that reported in [20] because it involves the Wood minima as well. The secondary array Wood minima has only little influence in the second order transmission peaks coming from the primary array, which are still flanked by primary Wood minima. From the point of view of the reciprocal space, the primary array is just a subset of the secondary array, thus the Wood anomaly minima of the primary array are also a subset of that of the secondary array. These features remain even when the secondary array is rectangular, as shows Fig. 5. The transmission dispersion for sample $\mathrm{C}$ is calculated following the path in the reciprocal space depicted in the insets of Fig. 5(a) and 5(c). When the Wood minima curves are added to the plot (Fig. 5(b) and (d)) it is clear that the interplay between Wood minima, resonance interference, and transmission peaks is more complex than for samples A or B.

Figure 6 represents the dependence of the transmission spectrum on the plate thickness. The compound array has $d_{1}=1 \mathrm{~mm}, d_{2}=4 \mathrm{~mm}$, and a $=10 \mathrm{~mm}$. Three different plate thicknesses are used in numerical calculations: $3 \mathrm{~mm}$ (Fig.6(a)), $5 \mathrm{~mm}$ (Fig.6(b)) and $10 \mathrm{~mm}$ (Fig.6(c)). First order Fabry-Perot resonances are behind both transmission peaks when the plate thickness is small, as showed in Fig.6(a). These peaks are shifted to larger wavelengths when we increase the plate thickness as in Fig. 6(b) with the addition of a new high transmission peak. If we further increase the plate thickness (Fig. 6(c)) only transmission peaks resulting from higher Fabry-Perot resonances are evident within the depicted wavelength range. Although similar to the behaviour found in simple hole arrays, this behaviour is more complex in compound hole arrays due to the interaction between resonances and existence of high transmission peaks even at wavelengths below the $\lambda=\mathrm{a}$ condition. 


\section{CONCLUSIONS}

Sound transmission through periodically perforated plates with compound periodic arrays of holes has been studied theoretically. A model in the rigid-solid limit to calculate transmission spectra and dispersion is presented. The transmission features of the compound array under study can be summarized as follows: a) in addition to the previously reported resonance interference minima due to the interaction between two Fabry-Perot resonances, Wood anomaly minima also play a role in this interaction for frequencies below the resonant transmission peak of the smaller array. b) For frequencies near and above that resonance, the effect of the Wood anomaly minima coming from the larger array in the resonant transmission maxima of the smaller array are negligible, i.e. peaks overcome dips. c) As a consequence of the above conclusions, and in contrast to simple hole array transmission, compound hole arrays allow the existence of transmission peaks at wavelengths well below $\lambda=a$ condition, where "a" is the compound array period.

We hope that this study could stimulate further investigation towards truly double periodic hole arrays in plates as well as applications as acoustic and ultrasonic filters.

\section{Acknowledgments}

This work has been supported by the Spanish MICINN (MAT2010-16879), Generalitat Valenciana (PROMETEO 2010/043) and Universidad Politecnica de Valencia (PAID06-10-1839). H. E. acknowledges the support of CSIC-JAEpredoc scholarship. 


\section{REFERENCES}

[1] T.W. Ebbesen, H. J. Lezec, H. F. Ghaemi, T. Thio and P.A. Wolf, Extraordinary optical transmission through sub-wavelength hole arrays Nature (London) 391 (1998) 667-669.

[2] J. A. Porto, F. J. Garcia-Vidal and J.B. Pendry, Transmission resonances on metallic gratings with very narrow slits, Phys. Rev. Lett. 83 (1999) 2845-2848.

[3] Y. Takakura, Optical resonance in a narrow slit in a thick metallic screen, Phys. Rev. Lett. 86 (2001), 5601-5604.

[4] Q. Cao and P. Lalanne, Negative role of surface plasmons in the transmission of metallic gratings with very narrow slits, Phys. Rev. Lett. 88, (2002), 057403057406.

[5] C. Genet and T. W. Ebbesen, Light in tiny holes, Nature (London) 445 (2007), 3942.

[6] J. Dintinger, I. Robel, P. V. Kamat, C. Genet and T. W. Ebbesen, Terahertz AllOptical Molecule- Plasmon Modulation, Adv. Mater., 18 (2006),1645-1648.

[7] J. Dintinger, S. Klein and T. W. Ebbesen, Molecule-Surface Plasmon Interactions in Hole Arrays: Enhanced Absorption, Refractive Index Changes, and All-Optical Switching, Adv. Mater., 18 (2006),1267-1270.

[8] F. J. Garcia de Abajo, Light scattering by particle and hole arrays, Rev. Mod. Phys. 79, (2007), 1267-1290.

[9] L. Martin-Moreno, F. J. Garcia-Vidal, H. J. Lezec, K. M. Pellerin, T. Thio, J. B. Pendry and T. W. Ebbesen, Theory of extraordinary optical transmission through subwavelength hole arrays, Phys. Rev. Lett. 86, (2001),1114-1117. 
[10] W. L. Barnes, A. Dereux and T. W. Ebbesen, Surface plasmon subwavelength optics, Nature (London), 424 (2003), 824-830.

[11] F.J. Garcia de Abajo, H. Estrada and F. Meseguer, Diacritical study of light, electrons and sound scattering by particles and holes, New J. Phys. 11, (2009), 093013.

[12] L. Zhou and G. A. Kriegsmann, Complete transmission through a periodically perforated rigid slab, J. Acoust. Soc. Am. 121 (2007), 3288-3299.

[13] M. Lu, X. Liu, L. Feng, J. Li, C. Huang, Y. Chen, Y. Zhu, S. Zhu and N. Ming, Extraordinary acoustic transmission through a 1D grating with very narrow apertures Phys. Rev. Lett. 99 (2007), 174301-174301.

[14] B. Hou, J. Mei, M. Ke, W. Wen, Z. Liu, J. Shi and P. Sheng, Tuning Fabry-Perot resonances via diffraction evanescent waves Phys. Rev. B 76 (2007), 054303054309

[15] J. Christensen, L. Martin-Moreno, F.J. Garcia-Vidal, Theory of resonant acoustic transmission through subwavelength apertures, Phys. Rev. Lett. 101, 014301, (2008) .

[16] H. Estrada, P. Candelas, A. Uris, F. Belmar, F. J. Garcia de Abajo, F. Meseguer, Extraordinary sound screening in perforated plates, Phys. Rev. Lett. 101 (8) (2008) 084302-084305.

[17] H. Estrada, P. Candelas, A. Uris, F. Belmar, F. Meseguer, F. J. Garcia de Abajo, Influence of the hole filling fraction on the ultrasonic transmission through plates with subwavelength aperture arrays, Appl. Phys.Lett. 93 (1) (2008) 011907011909. 
[18] H. Estrada, P. Candelas, A. Uris, F. Belmar, F. J. Garcia de Abajo, F. Meseguer, Influence of lattice symmetry on ultrasound transmission through plates with subwavelength aperture arrays, Appl. Phys. Lett. 95 (5) (2009) 051906-051906.

[19] H. Estrada, F. J. Garcia de Abajo, P. Candelas, A. Uris, F. Belmar, F. Meseguer, Angle-dependent ultrasonic transmission through plates with subwavelength hole arrays, Phys. Rev. Lett. 102 (14) (2009) 144301-144304.

[20] Z. Liu and G. Jin, Resonant acoustic transmission through compound subwavelength hole arrays: the role of phase resonances, J. Phys: Condens. Matter $21(2009) 445401$.

[21] H. Estrada, P. Candelas, A. Uris, F. Belmar, F. J. Garcia de Abajo, F. Meseguer, Sound Transmission through perforated plates with subwavelength hole arrays: A rigid-solid model, Wave Motion 48 (3) (2010), 235-242

[22] Charles Kittel. Introduction to Solid State Physics. John Wiley \& Sons, 7 th edition, 1996

[23] Milton Abramowitz and Irene A. Segun. Handbook of Mathematical Functions with Formulas, Graphs and Mathemathical Tables. Dover, New York.

[24] Earl G. Williams. Fourier Acoustics: sound radiation and nearfield acoustical holography. Academic Press, 1999.

[25] R. W. Wood, Phil. Mag. 4 (1902), 396. 


\section{FIGURES CAPTIONS}

Figure 1. Scheme of the (a) "xz" plane and (b) "xy" plane of the unit-cell. Gray regions correspond to the rigid solid whereas the surrounding fluid is divided in 3 regions as indicated by the labels in a). The vector $\left(\vec{r}-\vec{r}_{i}\right)_{\square}$ in (b) represents the projection over the $\mathrm{Z}=0$ plane of the vector defined from the centre of each hole to the appropriate points in the fluid region 2 .

Figure 2. Diagram showing compound hole arrays. The region inside the dashed line corresponds to the compound hole array unit cell. A primary square array (light gray) is combined with a larger secondary array (dark gray) having (a) square and (b) rectangular symmetry.

Figure 3. Transmitted sound power coefficient as a function of the wavelength for the primary (black) and secondary (gray) hole arrays calculated separately ((a) and (c)) and together ((b) and (d)) forming a compound hole array as depicted by the insets. In (a), (b) $d_{1}=2 \mathrm{~mm}, d_{2}=3 \mathrm{~mm}, \mathrm{a}=8 \mathrm{~mm}$, and $\mathrm{h}=3 \mathrm{~mm}$ (Sample A), whereas in (c), (d) $\mathrm{d}_{1}=2$ $\mathrm{mm}, \mathrm{d}_{2}=4 \mathrm{~mm}, \mathrm{a}=10 \mathrm{~mm}$, and $\mathrm{h}=5 \mathrm{~mm}$ (Sample B).

Figure 4. Transmitted sound power coefficient $\tau$ as a function of the normalized frequency $\mathrm{k}_{0} \mathrm{a} / \pi$ and the parallel-to-plate wavevector $\vec{Q}_{0}$ along the "M" and "X" directions of the irreducible Brillouin zone. In (b) and (d) Wood anomaly minima are added to the plot for the primary (white) and secondary (black) arrays. Sample A calculations are shown in (a) and (b), and sample B calculations in (c) and (d). 
Figure 5. Transmitted sound power coefficient tau as a function of the normalized frequency $\mathrm{k}_{0} \mathrm{a} / \pi$ and the parallel-to-plate wavevector $\vec{Q}$ along the directions of the first Brillouin zone showed in the left insets of (a) and (c) for sample C. In (b) and (d) Wood anomaly minima of the primary (white) and secondary (black) arrays are added to the plot.

Figure 6. Transmitted sound power coefficient as a function of the wavelength for $d_{1}=1$ $\mathrm{mm}, \mathrm{d}_{2}=4 \mathrm{~mm}, \mathrm{a}=10 \mathrm{~mm}$, and three different plate thicknesses, namely (a) $3 \mathrm{~mm}$, (b) 5 $\mathrm{mm}$, (c) $10 \mathrm{~mm}$. 


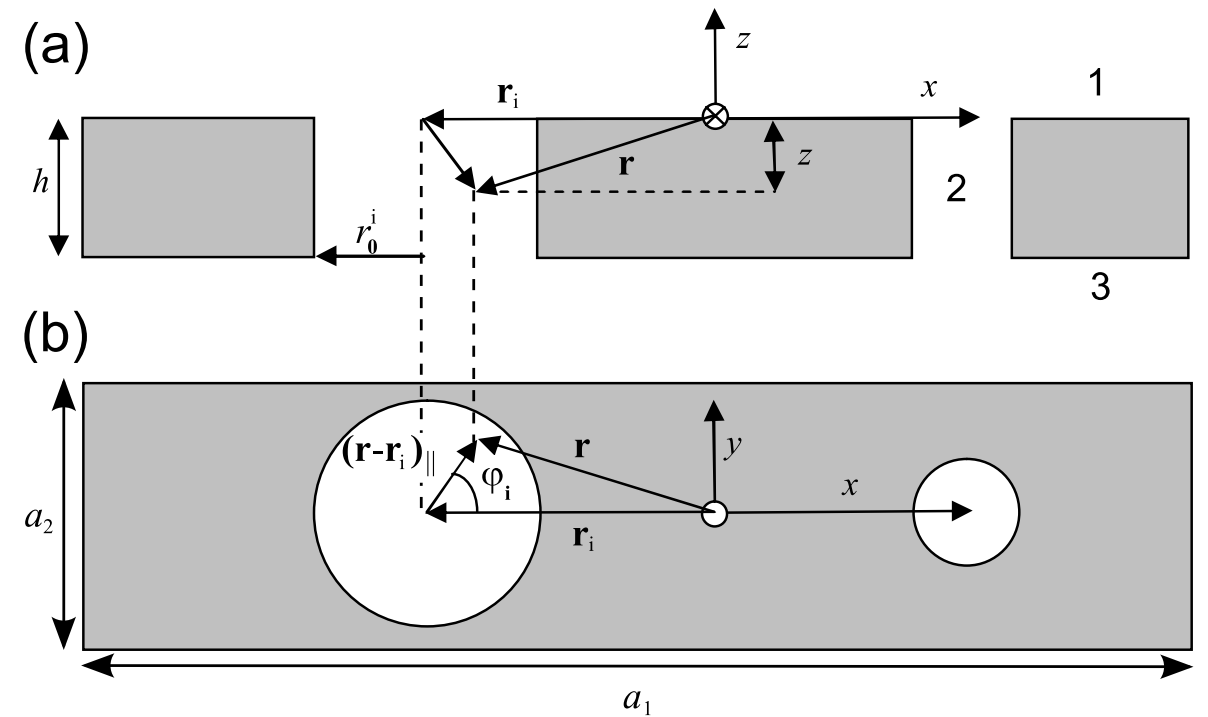

FIGURE 1 
(a)

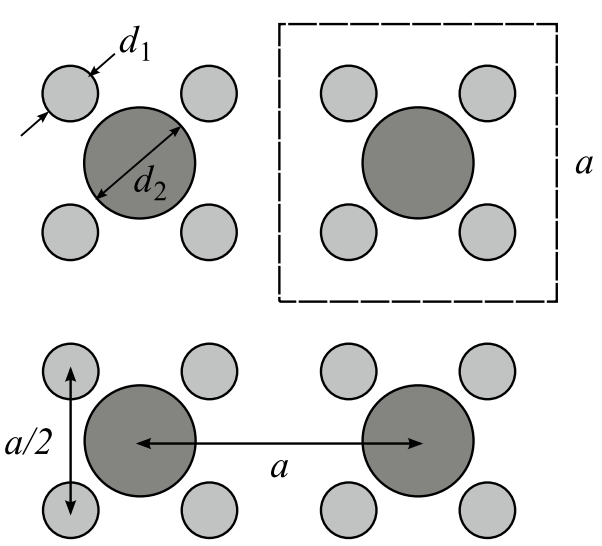

(b)

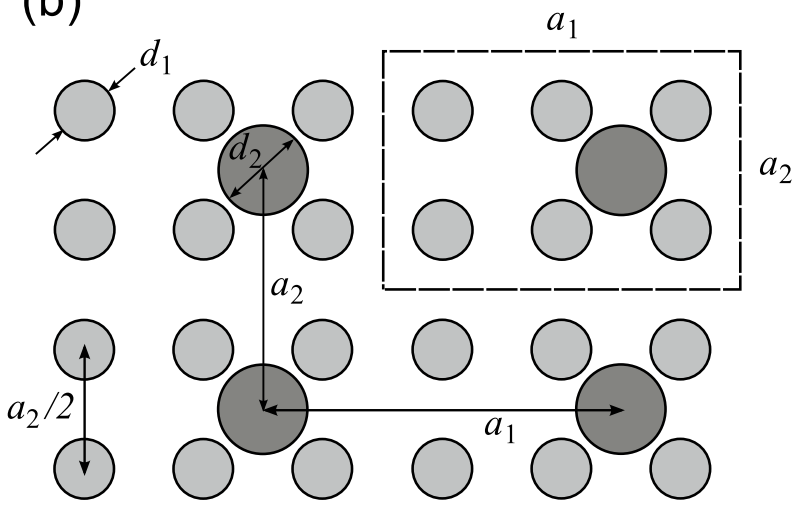

FIGURE 2 

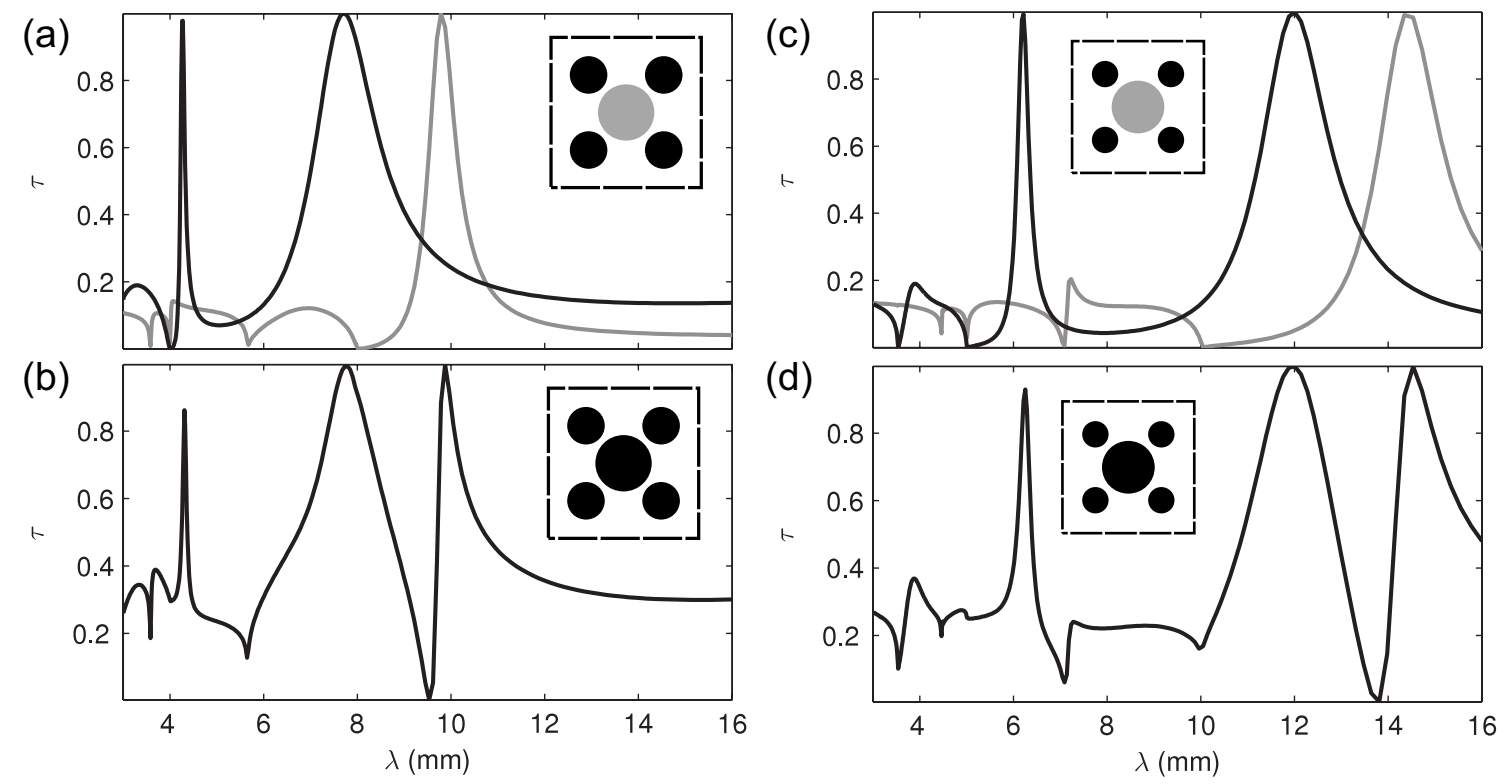

FIGURE 3 

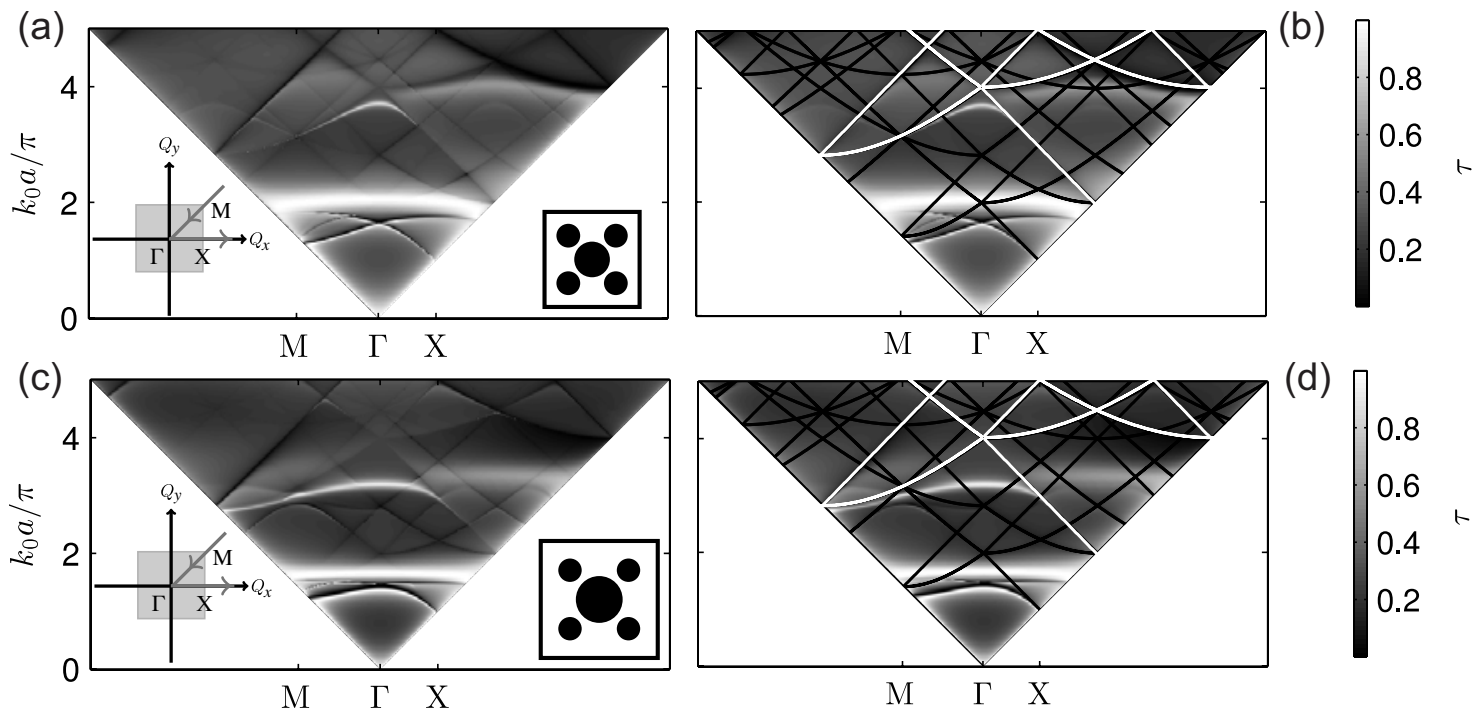

FIGURE 4 

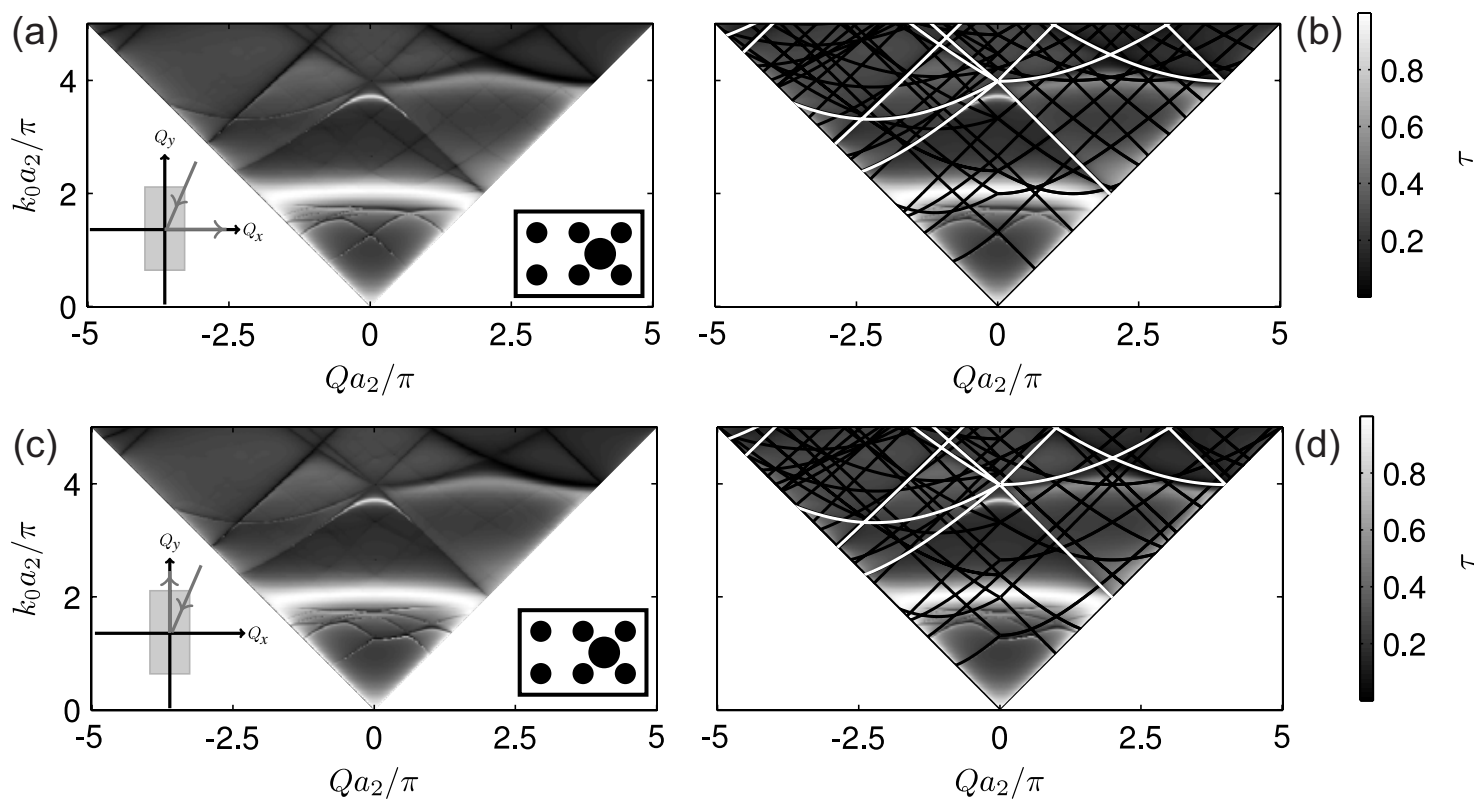

$\begin{cases}0.8 & \\ 0.6 & \\ 0.4 & \\ 0.2 & \end{cases}$

FIGURE 5 

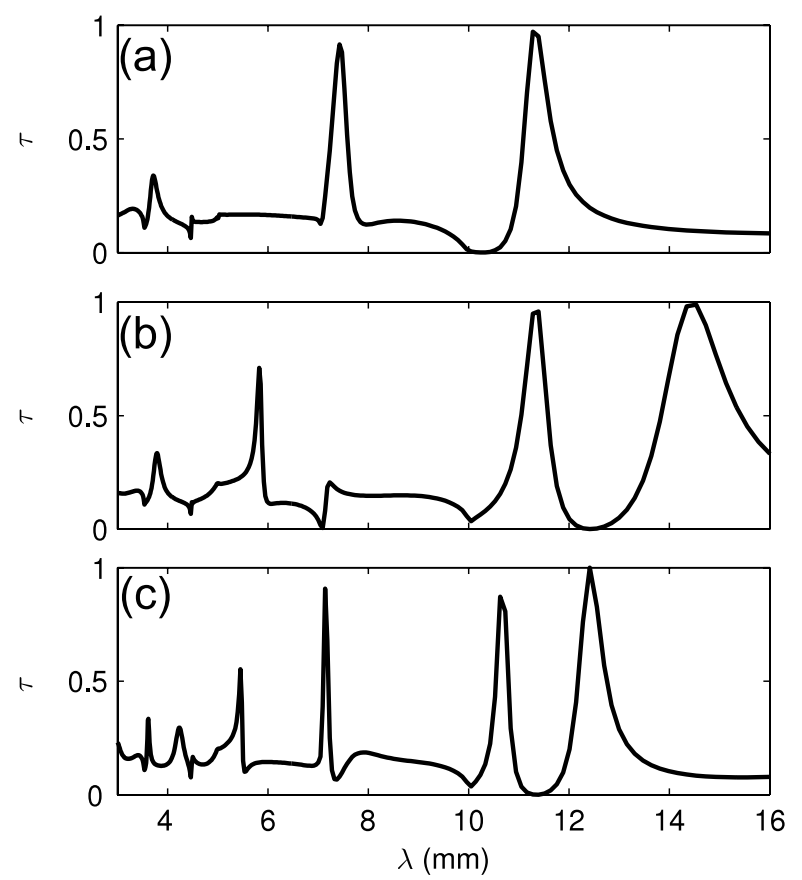

FIGURE 6 